\title{
Antifungal Resistance Pattern among Dermatophytes in Western Rajasthan
}

\author{
Prabhat Kiran Khatri ${ }^{1 *}$, Dileep Kachhawa ${ }^{1}$, Vinod Maurya ${ }^{1}$, Saroj Meena ${ }^{2}$, \\ Archana Bora ${ }^{2}$, Laxmi Rathore ${ }^{2}$, K.L. Seervi ${ }^{2}$ and Shivani Khullar ${ }^{2}$ \\ ${ }^{1}$ Department of Microbiology, ${ }^{2}$ Department of Skin and VD, Dr. S. N. Medical College, \\ Jodhpur, Rajasthan, India \\ *Corresponding author
}

\section{A B S T R A C T}

Keywords

Dermatophytosis,

Antifungal

Susceptibility

Testing, Disc

Diffusion Method,

Western

Rajasthan.

Article Info

Accepted:

04 June 2017

Available Online:

10 Julv 2017
Dermatophytosis is a major health related problem in areas with high humidity, overpopulation and poor hygienic conditions. Treatment options are topical as well as systemic antifungal drugs. But during course of time dermatophytes have also evolved drug resistance for single as well as multiple drugs simultaneously. Therefore it became necessary to find out antifungal susceptibility for dermatophytes. In this study we are using disc diffusion method for antifungal susceptibility testing. To find out antifungal resistance pattern among dermatophytes. 80 patients of all age group with clinical diagnosis of dermatophytosis were included under study. Skin, hair and nail samples were taken aseptically. These samples were subjected to $\mathrm{KOH}$ mount and culture on SDA. For sensitivity testing mature colony was subcultured on PDA and after significant sporulation, spores were harvested in normal saline and standardised inoculum of $1 \times 10^{6} \mathrm{cfu} / \mathrm{ml}$ was streaked over MHA plated and incubated at $28^{\circ} \mathrm{C}$. Zone of inhibition was measured after 37 days and were classified as sensitive, intermediate or resistant for a particular drug. Resistance against fluconazole and terbinafine was most common, $61.33 \%$ and $48 \%$ respectively. Resistance against voriconazole and miconazole was not observed in this study. Resistance against fluconazole was noted among all species of dermatophytes, followed by terbinafine and clotrimazole. Fluconazole was least effective drug followed by terbinafine. voriconazole and miconazole were more effective. Due to increasing trend of resistance among dermatophytes treatment should be based on antifungal sensitivity testing.

\section{Introduction}

Dermatophytes are filamentous fungi that are able to digest and obtain nutrients from keratin, primarily a component of skin hair and nails. Dermatophytes belong to three groups named as Trichophyton, Epidermophyton and Microsporum which can be further divided into anthropophilic, zoophilic and geophilic based on their natural habitat. Anthropophilic dermatophytes are associated with humans and rarely infect animals. Zoophilic dermatophytes cause infection in animals and may infect humans who come in contact. Geophilic dermatophytes are generally found in soil and take part in decomposition of hair, nails, feathers and horns (Ellis et al., 2000). When the organism grows on the host, living tissue is not usually invaded. The organism simply colonizes the keratinized outermost layer of skin. The disease is known as tinea or 
ringworm. It is the result of the host reaction to the enzymes released by the fungus during its digestive process. Dermatophytes are the only fungi that have evolved a dependency on human or animal infection for the survival of the species. It is therefore these fungi are among the most common infectious agents (Larone, 2002).

The tinea infections are prevalent worldwide but they are common in geographical areas with higher humidity. Overpopulation and poor hygienic living conditions also contribute to dermatophytic infections (vet al., 1995). Hot and humid climate of India makes Dermatophytosis a very common superficial fungal infection of skin (Niranjan et al., 2012). In recent years, the number of human infections caused by this group of fungi has increased considerably and is of particular concern in immune compromised patients (Walsh et al., 1999).

Studies conducted worldwide show that resistance among dermatophytes is not uncommon. Fernandez-torres et al., (2001) found fluconazole to be most resistant and voriconazole to be most sensitive drug for dermatophytes. Alfanso et al., (2005) found $5.6 \%$ resistant isolates among dermatophytes. In his study resistance against fluconazole was most common followed by itraconazole. Pakshir et al., (2009) noted $97.5 \%$ of dermatophytes isolate to be resistant to fluconazole and no resistance was noted for clotrimazole and miconazole. Azamabuja et al., (2013) found higher value of mic for fluconazole and itraconazole, $66.7 \%$ and $25 \%$ respectively. Jha et al., (2015) found fluconazole to be more resistant and terbinafine most effective antifungal.

A study conducted by Singal et al., 2001, in North India showed that there was treatment failure to griseofulvin among the Tinea capitis patients. Matnani et al., 2012, tested dermatophytes against terbinafine and found all isolates to be sensitive. Agarwal et al., 2015, found $11 \%$ strains resistant against fluconazole and $9 \%$ against terbinafine.

Due to high temperature and increased humidity, there are increased cases of dermatophytosis and other fungal infections especially in western Rajasthan. Since there was increased incidence of drug resistance observed over a period of time to the antimycotic drugs commonly used for the treatment i.e. fluconazole, terbinafine and clotrimazole. Therefore this study was carried out to find out incidence of dermatophytes infection as well as prevalence of drug resistance to fungal isolates. So far skin fungal infection is empirically treated and fungal culture and sensitivity is not routinely recommended in our region; therefore no data is available regarding incidence of skin fungal infection and drug resistance. Therefore this study was planned to find out the same.

\section{Materials and Methods}

\section{Study group}

Out of total tinea infected patients we selected only those patients who didn't respond to routine treatment, relapsed soon after stoppage of treatment or those having persistent dermatophytic infection. A total of eighty patients of all age group and both sexes attending skin outpatient department were recruited into the study.

\section{Sample collection}

All samples were collected from OPD of Department of Dermatology of our hospital, a tertiary care hospital catering western Rajasthan. This study was undertaken from September 2014 to November 2015. All the clinically suspected 80 cases were subjected to mycological work up. The specimens 
included skin scales, hair, and nails. The site of lesions were cleaned with $70 \%$ alcohol, samples were collected in a sterile paper folds and labelled with details of the patient. All the samples collected were subjected to direct microscopy and culture.

\section{Sample processing}

Direct microscopic examination of all samples was undertaken in $\mathrm{KOH}$ mount using $10 \%$ for skin and $40 \%$ for hair and Nail samples (Figure 1).

Samples were inoculated on slants of Sabouraud dextrose agar which contained chloramphenicol. Culture tubes were incubated at $28^{\circ} \mathrm{C}$ and were observed for growth. In our study a total of 75 isolates of dermatophytes out of 80 samples were isolated. Five samples out of 80 samples failed to grow in culture.

Identification of dermatophytes was done by studying colony morphology, obverse and reverse of colony surface (Figure 2), pigment production if any, and microscopic examination in lactophenol cotton blue mount (Figure 2).

Other biochemical tests like urease test and hair perforation test were also done if required.

In our hospital, routinely all patients with fungal infections attending OPD of dermatology, oral fluconazole $150 \mathrm{mg}$ is prescribed twice to thrice a week depending on area of fungal infection along with topical Clotrimazole or Miconazole cream. Those patients not responding to this treatment are prescribed terbinafine, itraconazole, griseofulvin or ketoconazole. Terbinafine is used systemically as well as topically. Griseofulvin is rarely used after introduction of safer drugs like fluconazole. Voriconazole is not available in our region.

\section{Antifungal susceptibility testing}

\section{Disc diffusion method}

The Agar based disc diffusion method was performed as described by Nweze et al., (2010) [14]. All dermatophytes were subcultured on potato dextrose agar and incubated at $28^{\circ} \mathrm{C}$ to enhance sporulation for one week. Following growth, conidia were harvested in sterile saline and conidial suspension was adjusted to $1 \times 10^{6}$ using hemocytometer. Eight Antifungal drugs were tested against dermatophyte isolates. Strength of drugs was, fluconazole $(25 \mu \mathrm{g})$, itraconazole $(10 \mu \mathrm{g})$, ketoconazole $(10 \mu \mathrm{g})$, clotrimazole $(10 \mu \mathrm{g})$, miconazole $(50 \mu \mathrm{g})$, voriconazole $(1 \mu \mathrm{g})$, terbinafine $(2 \mu \mathrm{g})$ and griseofulvin $(25 \mu \mathrm{g})$. Griseofulvin and terbinafine were not available commercially and were prepared in our labouratory. These two drugs were obtained in powdered form and stock solution of both drugs was prepared in dimethyl sulfoxide, as follows: griseofulvin $1.25 \mathrm{mg} / \mathrm{ml}$ and terbinafine $0.1 \mathrm{mg} / \mathrm{ml}$. Blank discs of $6 \mathrm{~mm}$ were loaded with $20 \mu \mathrm{l}$ of prepared stock solution to obtain the desired drug concentration per disc.

Plates of non-supplemented Mueller-Hinton Agar (MHA) were streaked evenly in three directions with a sterile cotton swab dipped into the standardized inoculums suspension. Plates were allowed to dry then antifungal discs were applied over MHA plates. Plates were inverted and incubated at $28^{\circ} \mathrm{C}$ and inhibition zone diameters were measured after 3-7 days for fungal growth (Nweze et al., 2010). Inhibition zone diameter was measured in millimeters and recorded as sensitive, intermediate or resistant (Table 1). Control plates with fungus inoculums and without antifungal discs were also tested. To evaluate reproducibility all tests were conducted in duplicate with new inoculums (Figure 3). 


\section{Quality control}

The reference isolates Aspergillus flavus ATCC 204304 on Mueller Hinton agar. Candida parapsilosis ATCC 22019 and Candida krusei ATCC 6258 were also included in each set of experiments for quality control on Mueller Hinton Agar supplemented with $2 \%$ glucose and $0.5 \mu \mathrm{g} / \mathrm{ml}$ of methylene blue (MHA-GMB) as specified in CLSI M44A (National Committee for Clinical Laboratory Standards, 2004).

\section{Results and Discussion}

In our study a total of 75 isolates of dermatophytes out of 80 samples were grown on culture. The isolates belonged to three genera and seven species as follows: Trichophyton mentagrophytes 31(41.3\%), Trichophyton rubrum 13(17.3\%), Trichophyton verrucosum 9(12\%), Trichophyton Tonsurans 12(16\%), Microsporum gypseum 5(6.7\%) and Epidermophyton floccosum 4(5.3\%), Trichophyton violaceum 1(1.3\%). T. mentagrophytes was most frequently isolated dermatophyte in our study.

Five isolates of $T$. rubrum (38.46\%) were resistant against fluconazole, four isolates $(30.77 \%)$ against terbinafine, five isolates $(38.46 \%)$ against clotrimazole and two isolates $(15.38 \%)$ were resistant against ketoconazole.

Twenty five isolates of $T$. mentagrophytes $(80.64 \%)$ were resistant against fluconazole, nineteen $(61.29 \%)$ against terbinafine, seven $(22.58 \%)$ against clotrimazole, three $(9.68 \%)$ against ketoconazole and three (9.68\%) against griseofulvin.

Six isolates of T. verrucosum (66.67\%) were resistant against fluconazole, seven (77.78\%) against terbinafine and two isolates (22.22\%) against clotrimazole. Seven isolates of $T$. tonsurans $(58.33 \%)$ were resistant against fluconazole, six (50\%) against terbinafine, five $(41.67 \%)$ against clotrimazole, four $(33.33 \%)$ resistant and two (16.6\%) intermediate against itraconazole and one isolate $(8.22 \%)$ against griseofulvin.

Two isolates of Microsporum gypseum (40\%) were resistant against fluconazole and three isolates $(60 \%)$ were intermediate against itraconazole.

Two isolates of Epidermophyton floccosum $(50 \%)$ were intermediate against fluconazole. No isolate of E. floccosum was found resistant against drugs tested.

T. violaceum was resistant against fluconazole only.

No isolate was found resistant against voriconazole and miconazole (Table 2).

After availability of antifungal sensitivity report prescription was revised. We changed the resistant drugs and switched them to one sensitive oral and one sensitive topical drug. 67 patients $(89.33 \%)$ out of 75 showed improvement and were cleared of infection after completion of treatment. Those patients who tested sensitive for all drugs or tested sensitive to those drugs that was already been prescribed, we took another sensitive drug or same drug at higher doses. These patients were also advised to maintain proper hygiene and were asked to keep the area dry, wear cotton clothes and avoid tight clothes. By doing all these measures these patients responded to treatment. Eight patients $(10.67 \%)$ didn't respond to all above measures and to change of drugs.

Dermatophytosis is a worldwide health related problem. The species of dermatophytes causing infection may vary 
from region to region. Some species are restricted to specific parts of world and some species like Trichophyton rubrum have a worldwide distribution (Gupta et al., 2003).

Different dermatophyte strains may have different antifungal susceptibility patterns which may vary geographically also. Some strains of dermatophytes resistant to particular antifungal agent have been reported (Nweze et al., 2010). Resistance against fluconazole and terbinafine is found very high, this may be due to easy availability and indiscriminate topical as well as systemic use of these antifungal drugs. Resistance against voriconazole is rare because of non- availability of this drug in this particular part of India.

Griseofulvin once a common antifungal, is least used now a days therefore less resistance against this drug is observed in our study. The introduction of wide range of newer antifungal agents and the isolation of clinical isolates showing resistance to azoles and allylamines group of antifungal drugs. This makes testing of the susceptibility of dermatophytes to these agents more important in treatment of resistant cases. This may also help in surveillance and epidemiological study of resistant strains.

Fig.1 KOH mount of skin scrapping showing fungal hyphae

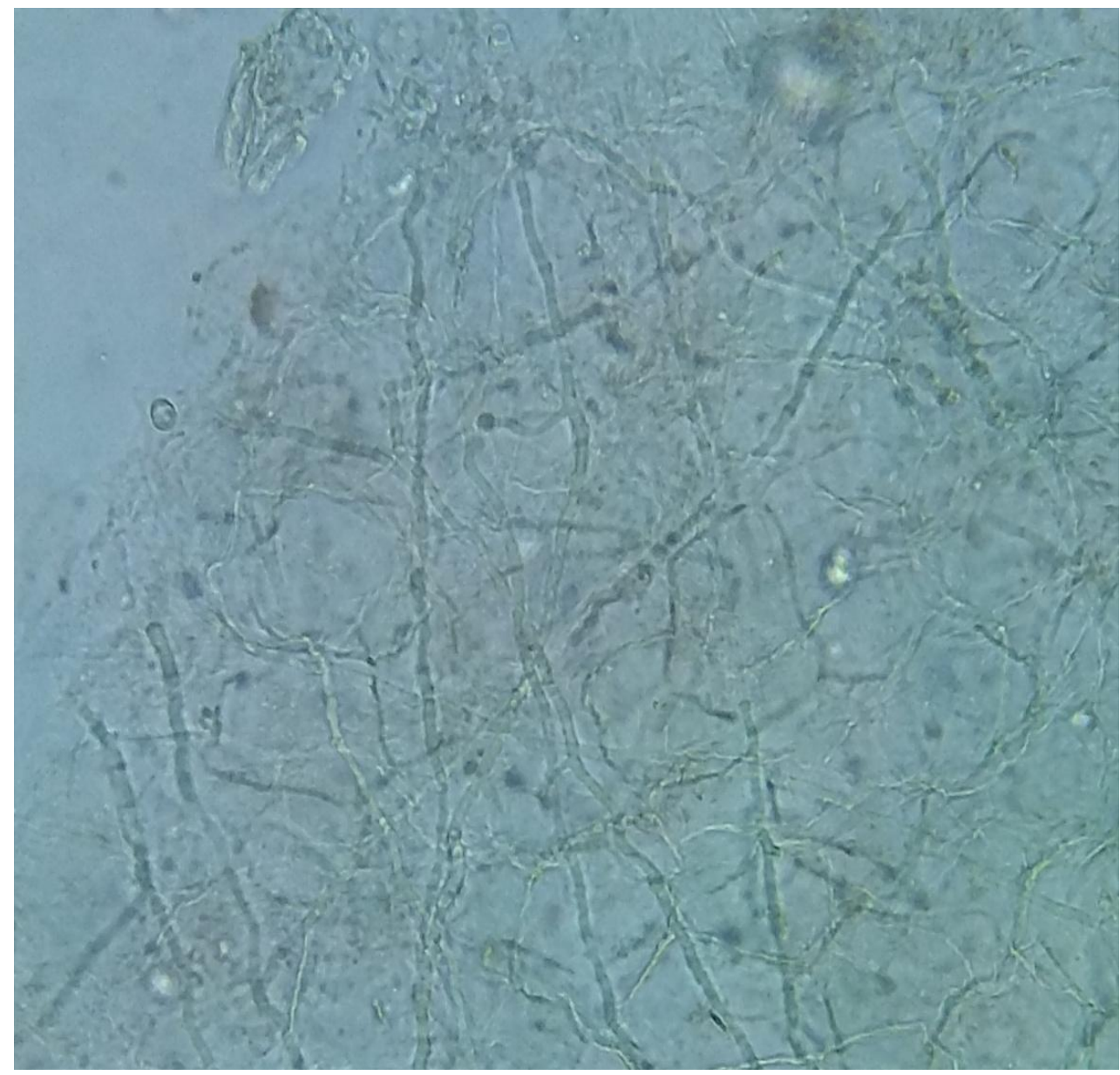


Fig.2 Diagram showing obverse and reverse of colony in upper part (a), and lactophenol cotton blue mount in lower part (b)

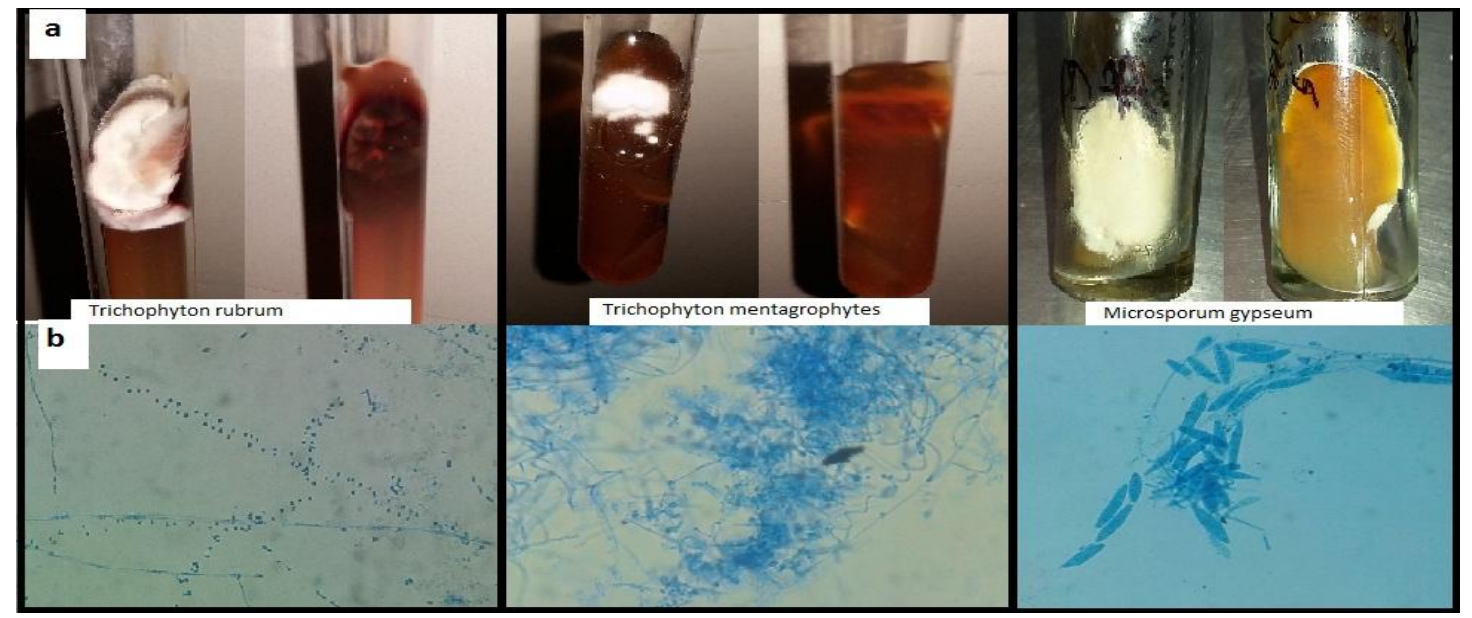

Fig.3 Antifungal susceptibility testing on Mueller-Hinton Agar plates showing zone of inhibition

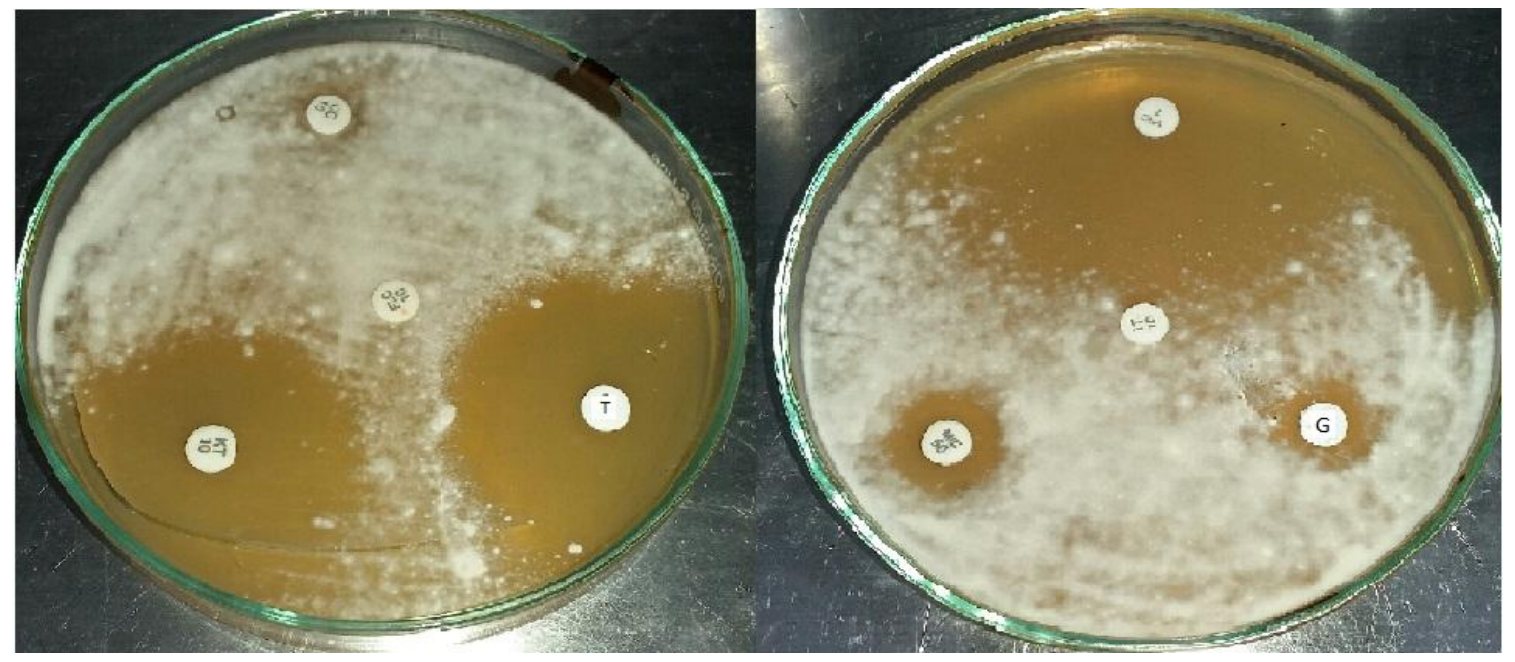

Table.1 Inhibition zone diameter criteria for susceptibility and

Resistance of antifungal discs (Keyvan et al., 2009; Agarwal et al., 2015; Galuppi et al., 2010)

\begin{tabular}{|l|c|c|c|c|}
\hline \multirow{2}{*}{$\begin{array}{c}\text { Antifungal } \\
\text { drugs }\end{array}$} & \multirow{2}{*}{ Potency } & \multicolumn{3}{c|}{ Zone diameter in mm } \\
\cline { 3 - 5 } & Sensitive & Intermediate & Resistant \\
\hline Fluconazole & $25 \mu \mathrm{g}$ & $\geq 20$ & $19-15$ & $\leq 14$ \\
\hline Voriconazole & $1 \mu \mathrm{g}$ & $>14$ & - & $<14$ \\
\hline Itraconazole & $10 \mu \mathrm{g}$ & $\geq 22$ & $21-15$ & $\leq 15$ \\
\hline Terbinafine & $2 \mu \mathrm{g}$ & $\geq 26$ & $26-20$ & $\leq 20$ \\
\hline Ketoconazole & $10 \mu \mathrm{g}$ & $\geq 30$ & $29-22$ & $\leq 22$ \\
\hline Miconazole & $50 \mu \mathrm{g}$ & $\geq 20$ & $19-11$ & $\leq 11$ \\
\hline Clotrimazole & $10 \mu \mathrm{g}$ & $\geq 20$ & $19-11$ & $\leq 11$ \\
\hline Griseofulvin & $25 \mu \mathrm{g}$ & $\geq 31$ & $26-31$ & $\leq 26$ \\
\hline
\end{tabular}


Table.2 Number of Dermatophyte Isolates against eight antifungal drugs tested

\begin{tabular}{|c|c|c|c|c|c|c|c|c|c|c|c|c|c|c|c|c|c|c|c|c|c|c|c|}
\hline \multirow{4}{*}{ Dermatophytes } & \multicolumn{23}{|c|}{ Number of dermatophyte isolates against respective IZD $(\mathbf{m m})$ of antifungal drugs } \\
\hline & \multicolumn{3}{|c|}{ FLU } & \multicolumn{2}{|c|}{ VOR } & \multicolumn{3}{|c|}{ ITR } & \multicolumn{3}{|c|}{ TER } & \multicolumn{3}{|c|}{ KET } & \multicolumn{3}{|c|}{ MIC } & \multicolumn{3}{|c|}{ CLO } & \multicolumn{3}{|c|}{ GRI } \\
\hline & $\mathbf{S}$ & I & $\mathbf{R}$ & $\mathbf{S}$ & $\mathbf{R}$ & $\mathbf{S}$ & I & $\mathbf{R}$ & $\mathbf{S}$ & I & $\mathbf{R}$ & $\mathbf{S}$ & $\mathbf{I}$ & $\mathbf{R}$ & $\mathbf{S}$ & $\mathbf{I}$ & $\mathbf{R}$ & $\mathbf{S}$ & $\mathbf{I}$ & $\mathbf{R}$ & $\mathbf{S}$ & $\mathbf{I}$ & $\mathbf{R}$ \\
\hline & $\geq 20$ & $\begin{array}{c}19- \\
15 \\
\end{array}$ & $\leq 14$ & $>14$ & $<14$ & $\geq 22$ & $\begin{array}{c}21- \\
15 \\
\end{array}$ & $\leq 15$ & $\geq 26$ & $\begin{array}{c}26- \\
20 \\
\end{array}$ & $\leq 20$ & $\geq 30$ & $\begin{array}{l}29- \\
22 \\
\end{array}$ & $\leq 22$ & $\geq 20$ & $\begin{array}{c}19- \\
11\end{array}$ & $\leq 11$ & $\geq 20$ & $\begin{array}{c}19- \\
11\end{array}$ & $\leq 11$ & $\geq 31$ & $\begin{array}{c}26- \\
31 \\
\end{array}$ & $\leq 26$ \\
\hline $\begin{array}{l}\text { T. rubrum } \\
(N=13)\end{array}$ & 8 & 0 & 5 & 13 & 0 & 13 & 0 & 0 & 9 & 0 & 4 & 11 & 0 & 2 & 13 & 0 & 0 & 8 & 0 & 5 & 13 & 0 & 0 \\
\hline $\begin{array}{l}\text { T. mentagrophytes } \\
(N=31)\end{array}$ & 6 & 0 & 25 & 31 & 0 & 31 & 0 & 0 & 12 & 0 & 19 & 28 & 0 & 3 & 31 & 0 & 0 & 24 & 0 & 7 & 28 & 0 & 3 \\
\hline $\begin{array}{l}\text { T. verrucosum } \\
(N=9)\end{array}$ & 3 & 0 & 6 & 9 & 0 & 9 & 0 & 0 & 2 & 0 & 7 & 9 & 0 & 0 & 9 & 0 & 0 & 7 & 0 & 2 & 9 & 0 & 0 \\
\hline $\begin{array}{l}\text { T. tonsurans } \\
(N=12)\end{array}$ & 5 & 0 & 7 & 12 & 0 & 6 & 2 & 4 & 6 & 0 & 6 & 12 & 0 & 0 & 12 & 0 & 0 & 7 & 0 & 5 & 11 & 0 & 1 \\
\hline $\begin{array}{l}\text { M. gypseum } \\
(N=5)\end{array}$ & 3 & 0 & 2 & 5 & 0 & 2 & 3 & 0 & 5 & 0 & 0 & 5 & 0 & 0 & 5 & 0 & 0 & 5 & 0 & 0 & 5 & 0 & 0 \\
\hline $\begin{array}{l}\text { E. floccosum } \\
(N=4)\end{array}$ & 2 & 2 & 0 & 4 & 0 & 4 & 0 & 0 & 4 & 0 & 0 & 4 & 0 & 0 & 4 & 0 & 0 & 4 & 0 & 0 & 4 & 0 & 0 \\
\hline $\begin{array}{l}\text { T. violaceum } \\
(N=1)\end{array}$ & 0 & 0 & 1 & 1 & 0 & 1 & 0 & 0 & 1 & 0 & 0 & 1 & 0 & 0 & 1 & 0 & 0 & 1 & 0 & 0 & 1 & 0 & 0 \\
\hline $\begin{array}{c}\text { Total } \\
(\mathrm{N}=75)\end{array}$ & 27 & 2 & 46 & 75 & 0 & 66 & 5 & 4 & 39 & 0 & 36 & 70 & 0 & 5 & 75 & 0 & 0 & 56 & 0 & 19 & 71 & 0 & 4 \\
\hline
\end{tabular}

$\mathrm{S}$ - no. of sensitive isolates, R- no. of resistant isolates, I- no. of intermediate isolates. FLU: fluconazole, VOR: voriconazole, ITR: itraconazole, TER: terbinafine, KET: ketoconazole, MIC: miconazole, CLO: clotrimazole, GRI: griseofulvin, IZD: inhibition zone diameter. 
Drug resistance mechanism among dermatophytes are acquired by stress adaptation against fluconazole, and terbinafine; modification of squalene epoxidase enzyme by mutation, overexpression of salicylate mono-oxygenase (drug degradation) against terbinafine; over expression of lanosterol-14a- demethylase and drug efflux against ketoconazole; drug efflux is the mechanism that is tool of resistance against all antifungal drugs by dermatophytes (Martinez et al., 2008). Biofilm formation has also been reported as a mechanism of drum resistance by $T$. Rubrum and T. mentagrophytes (Costa et al., 2014). Among these mechanisms, increased drug efflux is most common method of development of resistance.

Some researchers have conducted the susceptibility testing of dermatophytes by using broth microdilution method (Esteban et al., 2005; Barchiesi et al., 2001; Favre et al., 2003; Fernández et al., 2003) based on CLSI M38-A 2002 protocol (National Committee for Clinical Laboratory Standards, 2002), but this method was for filamentous fungi other than dermatophytes. Later, the document was modified to M38-A2 by CLSI in 2008. This document also includes the protocol for dermatophytes. This updated method was used by some authors to find MIC against antifungal drugs for different species of dermatophytes.

Although no simple reference method has been standardized for testing of drug susceptibility for dermatophytes by disc diffusion method, some authors have formulated simple agar based disc diffusion methods using SDA mediu (Esteban et al., 2005), Mueller-Hinton Agar medium supplemented with $2 \%$ glucose and $0.5 \mu \mathrm{g} / \mathrm{ml}$ Methylene blue as per CLSI M44-A (Diogo et al., 2010; Castro et al., 2008), Mueller-Hinton Agar medium, RPMI 1640 supplemented with
$2 \%$ glucose and 1.5 to $1.8 \%$ agar (Castro et al., 2008; Aktas et al., 2014), RPMI 1640 with 1.5\%agar (Galuppi et al., 2010) and also using some other media (Fernández et al., 2006; Singh et al., 2007). We used MuellerHinton agar media which is easily available in laboratory, economical and easy to perform test. In our study 75 dermatophyte isolates from skin, hair and nails samples were tested against eight antifungal drugs by using disc diffusion method.

Among oral antifungal agents resistance against fluconazole was most common $(61.33 \%)$ in our study. High fluconazole resistance has also been reported by some other workers (Galuppi et al., 2010). Resistance against itraconazole was not very common in this study, only four isolates $(5.33 \%)$ of $\mathrm{T}$. tonsurans were found resistant and five isolates $(6.67 \%)$ were intermediate. Similar findings have also been reported by some authors. Resistance against griseofulvin was uncommon. Our study has also found low resistance against griseofulvin (5.33\%). Most of the authors have not found resistance but some authors have reported low resistance against griseofulvin. Resistance against ketoconazole $(6.67 \%)$ was also found in $T$. rubrum and $T$. mentagrophytes which is similar to other studies (Aktas et al., 2014). Voriconazole which is unavailable in our region, resistance to this was not observed in our study $(0 \%)$ which is similar with several other studies (Castro et al., 2008), but some authors have found very small percentage of resistance against voriconazole (Carrillo et al., 2005).

Among topical antifungal agents resistance against clotrimazole was also common $(25.33 \%)$ in our study and also reported by some authors (Keyvan et al., 2009), but some studies have not found resistance against clotrimazole. Resistance against miconazole was not observed $(0 \%)$ in our study which is 
similar to other studies conducted by few authors (Nweze et al., 2010). Terbinafine which is used systemically as well as topically, resistance against it was also very high $(48 \%)$ and have also been reported by other authors like our study (Mukherjee et al., 2003).

Some authors from India have also reported resistance among dermatophytes. Agarwal et al., (2015) reported $11 \%$ resistance against fluconazole and 9\% resistance against terbinafine. Singal et al., noted treatment failure with griseofulvin treatment. Matnani et al., noted excellent activity of terbinafine against dermatophytes. In his study all dermatophytes were found sensitive against terbinafine. Bhatia et al., (2015) observer higher MIC values for terbinafine by $T$. mentagrophytes and T. rubrum isolates.

The present study showed that resistance against fluconazole is most common followed by terbinafine and clotrimazole. In our study miconazole and voriconazole seems to be better drugs followed by ketoconazole and itraconazole. Because of low cost and easily availability miconazole seems to be better topical antifungal agent for dermatophytes. Voriconazole followed by griseofulvin and itraconazole seems to be better oral antifungal drugs.

As antifungal susceptibility testing facilities are now available for dermatophytes, every isolate should be tested against antifungal drugs so that increasing resistance among dermatophytes can be reduced.

Few studies have not found consistent correlation between disc diffusion method and broth micro-dilution method still agar plate method give reproducible results and is easy to perform, therefore this method can be used as a screening method for detection of resistance among dermatophytes.

\section{References}

Agarwal, R.K., Gupta, S., Mittal, G., Khan, F., Roy, S., Agarwal, A. 2015. Antifungal Susceptibility Testing of Dermatophytes by Agar Based Disk Diffusion Method. Int. J. Curr. Microbiol. App. Sci., 4(3): 430-6.

Aktas, A.E., Yigit, N., Aktas, A., Gozubuyuk, S.G. 2014. Investigation of In Vitro Activity of Five Antifungal Drugs against Dermatophytes Species Isolated from Clinical Samples Using the E-Test Method. Eurasian J. Med., 46(1): 26.

Azambuja, C.V., Pimmel, L.A., Klafke, G.B., Xavier, M.O. 2014. Onychomycosis: Clinical, mycological and in vitro susceptibility testing of isolates of Trichophyton rubrum. An. Bras. Dermatol., 89: 581-6.

Barchiesi, F., Arzeni, D., Camiletti, V., Simonetti, O., Cellini, A., Offidani, A.M., Scalise, G. 2001. In vitro activity of posaconazole against clinical isolates of dermatophytes. J. Clin. Microbiol., 39(11): 4208-9.

Bhatia, V.K., Sharma, P.C. 2015. Determination of minimum inhibitory concentrations of itraconazole, terbinafine and ketoconazole against dermatophyte species by broth microdilution method. Indian J. Med. Microbiol., 33(4): 533.

Carrillo-Muñoz, A.J., Cárdenes, C.D., Carrillo-Orive, B., Rodríguez, V., del Valle, O., Casals, J.B., Ezkurra, P.A., Quindós, G. 2005. In vitro antifungal activity of voriconazole against dermatophytes and superficial isolates of Scopulariopsis brevicaulis. Revistaiberoamericana de micología, 22(2): 110 .

Castro Méndez, C., Carmen Serrano, M., Valverde, A., Pemán, J., Almeida, C., Martín-Mazuelos, E. 2008. Comparison of E-Test ${ }^{\circledR}$, disk diffusion and a 
modified CLSI broth microdilution (M 38-A) method for in vitro testing of itraconazole, fluconazole and voriconazole against dermatophytes. Med. Mycol., 46(2): 119-23.

Chadeganipour, M., Nilipour, S., Havaei, A. 2004. In vitro evaluation of griseofulvin against clinical isolates of dermatophytes from Isfahan. Mycoses, 47(11-12): 503-7.

CLSI. 2008. Reference Method for Broth Dilution Antifungal Susceptibility Testing of Filamentous Fungi; Approved Standard - Document M38A2. 2nd ed. Wayne, PA: Clinical and Laboratory Standards Institute.

Costa Orlandi, C.B., Sardi, J.C., Santos, C.T., FuscoAlmeida, A.M., MendesGiannini, M.J. 2014. In vitro characterization of Trichophyton rubrum and $T$. mentagrophytes biofilms. Biofouling, 30: 719-27.

Diogo, H.C., Melhem, M., Sarpieri, A., Pires, M.C. 2010. Evaluation of the diskdiffusion method to determine the in vitro efficacy of terbinafine against subcutaneous and superficial mycoses agents. Anaisbrasileiros de dermatologia, 85(3): 324-30.

Ellis, D., Marriott, D., Hajjeh, R.A., Warnock, D., Meyer, W., Barton, R. 2000. Epidemiology: surveillance of fungal infections. Med. Mycol., 38(sup1): 17382.

Esteban, A., Abarca, M.L., Cabanes, F.J. 2005. Comparison of disk diffusion method and broth microdilution method for antifungal susceptibility testing of dermatophytes. Med. Mycol., 43(1): 616.

Favre, B., Hofbauer, B., Hildering, K.S., Ryder, N.S. 2003. Comparison of in vitro activities of 17 antifungal drugs against a panel of 20 dermatophytes by using a microdilution assay. J. Clin. Microbiol., 41(10): 4817-9.
Fernández-Torres, B., Cabanes, F.J., CarrilloMunoz, A.J., Esteban, A., Inza, I., Abarca, L., Guarro, J. 2002. Collaborative evaluation of optimal antifungal susceptibility testing conditions for dermatophytes. J. Clin. Microbiol., 40(11): 3999-4003.

Fernández-Torres, B., Carrillo, A.J., Martın, E., Del Palacio, A., Moore, M.K., Valverde, A., Serrano, M., Guarro, J. 2001. In vitro activities of 10 antifungal drugs against 508 dermatophyte strains. Antimicrob. Agents and Chemother., 45(9): 2524-8.

Fernández-Torres, B., Carrillo-Muñoz, A., Inza, I., Guarro, J. 2006. Effect of culture medium on the disk diffusion method for determining antifungal susceptibilities of dermatophytes. Antimicrob Agents and Chemother., 50(6): 2222-4.

Fernández-Torres, B., Inza, I., Guarro, J. 2003. In vitro activities of the new antifungal drug eberconazole and three other topical agents against 200 strains of dermatophytes. J. Clin. Microbiol., 41(11): 5209-11.

Galuppi, R., Gambarara, A., Bonoli, C., Ostanello, F., Tampieri, M.P. 2010. Antimycotic effectiveness against dermatophytes: comparison of two in vitro tests. Vet. Res. Communications, 34(1): 57-61.

Gupta, A.K., Kohli, Y. 2003. In vitro susceptibility testing of ciclopirox, terbinafine, ketoconazole and itraconazole against dermatophytes and nondermatophytes, and in vitro evaluation of combination antifungal activity. Br. J. Dermatol., 149(2): 296305.

Jha, B.K., Mahadevamurthy, S., Sudisha, J., Bora, A. 2015. Isolation, Identification and Antifungal Susceptibility Test of Dermatophytes from the Patients with Onychomycosis in Central Nepal. 
American J. Dermatol. Venereol., 4(3): 30-6.

Keyvan, P., Leila, B., Zahra, R., Manuchehr, S., Kamiar, Z. 2009. In vitro activity of six antifungal drugs against clinically important dermatophytes. Jundishapur J. Microbiol., 158-63.

Larone, D.H. 2002. Dermatophytes. In: medically important fungi - Aguide to identification. 4th Edn; ASM Press, Washington DC, 241-253.

Martinez-Rossi, N.M., Peres, N.T., Rossi, A. 2008. Antifungal resistance mechanisms in dermatophytes. Mycopathologia, 166(5-6): 369-83.

Matnani, G., Roy, I., Gandham, N., Ujagare, M., Jadhav, S.V. Identification and Antifungal Susceptibility Testing of Fungal Infections in Clinical Samples of Suspected Superficial Fungal Infections. Bioinfo Publications, 3(7): 215-220.

Mukherjee, P.K., Leidich, S.D., Isham, N., Leitner, I., Ryder, N.S., Ghannoum, M.A. 2003. Clinical Trichophyton rubrum strain exhibiting primary resistance to terbinafine. Antimicrob. Agents Chemother., 47(1): 82-6.

National Committee for Clinical Laboratory Standards. 2002. Reference Method for Broth Dilution Antifungal Susceptibility Testing of Filamentous Fungi. Approved Standard M38-A. Wayne, PA: National Committee for Clinical Laboratory Standards.
National Committee for Clinical Laboratory Standards. 2004. Method for Antifungal Disk Diffusion Susceptibility Testing of Yeasts: Approved Guideline M44A.Wayne, PA, USA: NCCLS.

Niranjan, H.P., Padmaja, N., Priyanka, B.V. 2012. Study of onychomycosis at a tertiary care hospital in South India. $J$. Evol. Med. Dent. Sci., 1(5): 823-829.

Nweze, E.I., Mukherjee, P.K., Ghannoum, M.A. 2010. Agar-based disk diffusion assay for susceptibility testing of dermatophytes. J. Clin. Microbiol., 48(10): 3750-2.

Singal, A., Rawat, S., Bhattacharya, S.N., Mohanty, S., Baruah, M.C. 2001. Clinico- myocological profile of tinea $^{-}$ capitis in North India and response to griseofulvin. J. Dermatol., 28: 22-6.

Singh, J., Singh, J., Zaman, M., Gupta, A.K. 2007. Evaluation of microdilution and disk diffusion methods for antifungal susceptibility testing of dermatophytes. Med. Mycol., 45(7): 595-602.

Walsh, T.J., Groll, A.H. 1999. Emerging fungal pathogens: evolving challenges to immunocompromised patients for the twenty- first century. Transplant Infect. Dis., 1(4): 247-61.

Weitzman, I., Summerbell, R.C. 1995. The dermatophytes. Clin. Microbiol. Rev., 8(2): $240-59$.

\section{How to cite this article:}

Prabhat Kiran Khatri, Dileep Kachhawa, Vinod Maurya, Saroj Meena, Archana Bora, Laxmi Rathore, K.L. Seervi and Shivani Khullar. 2017. Antifungal Resistance Pattern among Dermatophytes in Western Rajasthan. Int.J.Curr.Microbiol.App.Sci. 6(7): 499-509. doi: https://doi.org/10.20546/ijcmas.2017.607.060 